\title{
Giant Outer Transiting Exoplanet Mass (GOT 'EM) Survey. I. Confirmation of an Eccentric, Cool Jupiter with an Interior Earth-sized Planet Orbiting Kepler-1514*
}

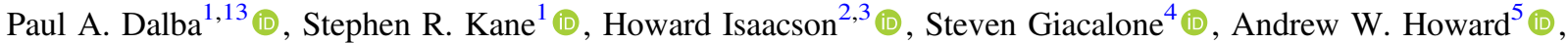

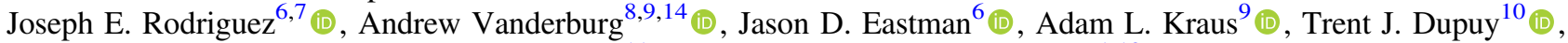 \\ Lauren M. Weiss ${ }^{11}$ (D), and Edward W. Schwieterman ${ }^{1,12}$ (iD \\ ${ }^{1}$ Department of Earth and Planetary Sciences, University of California Riverside, 900 University Ave., Riverside, CA 92521, USA; pdalba@ucr.edu \\ ${ }_{2}^{2}$ Department of Astronomy, University of California, Berkeley, Berkeley, CA 94720, USA \\ ${ }^{3}$ Centre for Astrophysics, University of Southern Queensland, Toowoomba, QLD, Australia \\ ${ }^{4}$ Department of Astronomy, University of California, Berkeley, Berkeley, CA 94720-3411, USA \\ 5 Department of Astronomy, California Institute of Technology, Pasadena, CA 91125, USA \\ ${ }^{6}$ Center for Astrophysics | Harvard \& Smithsonian, 60 Garden St., Cambridge, MA 02138, USA \\ ${ }^{7}$ Department of Physics and Astronomy, Michigan State University, East Lansing, MI 48824, USA \\ ${ }^{8}$ Department of Astronomy, University of Wisconsin-Madison, Madison, WI 53706, USA \\ ${ }^{9}$ Department of Astronomy, The University of Texas at Austin, Austin, TX 78712, USA \\ ${ }^{10}$ Institute for Astronomy, University of Edinburgh, Royal Observatory, Blackford Hill, Edinburgh, EH9 3HJ, UK \\ ${ }^{11}$ Institute for Astronomy, University of Hawai 'i, 2680 Woodlawn Drive, Honolulu, HI 96822, USA \\ ${ }^{12}$ Blue Marble Space Institute of Science, Seattle, WA 98115, USA \\ Received 2020 October 23; revised 2020 December 8; accepted 2020 December 14; published 2021 February 4
}

\begin{abstract}
Despite the severe bias of the transit method of exoplanet discovery toward short orbital periods, a modest sample of transiting exoplanets with orbital periods greater than 100 days is known. Long-term radial velocity (RV) surveys are pivotal to confirming these signals and generating a set of planetary masses and densities for planets receiving moderate to low irradiation from their host stars. Here we conduct RV observations of Kepler-1514 from the Keck I telescope using the High Resolution Echelle Spectrometer. From these data, we measure the mass of the statistically validated giant $\left(1.108 \pm 0.023 R_{\mathrm{J}}\right)$ exoplanet Kepler-1514 b with a 218 -day orbital period as $5.28 \pm 0.22 M_{\mathrm{J}}$. The bulk density of this cool $(\sim 390 \mathrm{~K})$ giant planet is $4.82_{-0.25}^{+0.26} \mathrm{~g} \mathrm{~cm}^{-3}$, consistent with a core supported by electron degeneracy pressure. We also infer an orbital eccentricity of $0.401_{-0.014}^{+0.013}$ from the RV and transit observations, which is consistent with planet-planet scattering and disk cavity migration models. The Kepler-1514 system contains an Earth-size, Kepler Object of Interest on a 10.5-day orbit that we statistically validate against false-positive scenarios, including those involving a neighboring star. The combination of the brightness $(V=11.8)$ of the host star and the long period, low irradiation, and high density of Kepler-1514 b places this system among a rare group of known exoplanetary systems and as one that is amenable to continued study.

Unified Astronomy Thesaurus concepts: Radial velocity (1332); Exoplanets (498); Direct imaging (387); Transit photometry (1709); Extrasolar gaseous giant planets (509)
\end{abstract}

\section{Introduction}

The transit method is not conducive to the discovery of planets with orbital distances like those of the solar system planets. The probability of observing an exoplanet transit scales inversely with the star-planet separation due to geometry, from the random orientation of orbital inclinations, and sampling, from the limited baseline of continuous observations from transit surveys (Beatty \& Gaudi 2008). These factors have combined to largely exclude planets with orbital periods $(P)$ greater than 100 days from the list of known transiting exoplanets.

The short-period bias of the transit method has a direct effect on the scientific return of observational investigations of exoplanets. The favorable geometry of a transit enables a suite of novel characterization techniques, most notably transmission spectroscopy (e.g., Seager \& Sasselov 2000). This technique has powered a thriving discipline of atmospheric characterization for short-period,

\footnotetext{
* Some of the data presented herein were obtained at the W. M. Keck Observatory, which is operated as a scientific partnership among the California Institute of Technology, the University of California, and the National Aeronautics and Space Administration. The Observatory was made possible by the generous financial support of the W. M. Keck Foundation.

13 NSF Astronomy and Astrophysics Postdoctoral Fellow.

14 NASA Sagan Fellow.
}

close-in exoplanets (e.g., Sing et al. 2016; Deming \& Seager 2017; Wakeford et al. 2017; Madhusudhan 2019; Welbanks et al. 2019). Similar observations, but of exoplanets on wider orbits with cooler temperatures, would be equally as transformative and would enable new comparative studies between exoplanets and the solar system. Indeed, simulated observations of exoplanet analogs of the solar system giant planets have found an amenability to transmission spectroscopy (Irwin et al. 2014; Dalba et al. 2015), as well as the novel technique of out-of-transit atmospheric characterization via refracted starlight (Sidis \& Sari 2010; Dalba 2017; Alp \& Demory 2018).

Efforts to discover and maintain the ephemerides of long-period (roughly $P \gtrsim 100$ days) transiting exoplanets have been underway for years. Some planets, like HD 80606 b, were first identified in radial velocity (RV) observations (Naef et al. 2001) and were later found to have a transiting geometry (Laughlin et al. 2009; Moutou et al. 2009). However, this happy coincidence is expected to be quite rare (Dalba et al. 2019). The vast majority of known longperiod transiting exoplanets were identified through dedicated transit surveys. The constraints of ground-based observations have limited orbital periods of transiting exoplanets to less than roughly 25 days (e.g., Brahm et al. 2016; Dittmann et al. 2017). From space, where observational baselines are far less limited, a variety 
of exoplanets with orbital periods greater than approximately 100 days have been found.

Data from the primary Kepler mission (Borucki et al. 2010; Thompson et al. 2018)— the longest continuous baseline transit survey conducted to date-have been meticulously searched for transits of long-period planets (Wang et al. 2015; Morton et al. 2016; Uehara et al. 2016; Kawahara \& Masuda 2019). Related efforts have not only produced catalogs of objects with orbital periods between 100 and 1000 days but also revealed information about their underlying populations (Foreman-Mackey et al. 2016; Herman et al. 2019) and the likelihood of finding additional planets in their systems (Dalba \& Muirhead 2016; Dalba \& Tamburo 2019; Masuda et al. 2020). A subset of Kepler's longestperiod transiting planets are circumbinary (e.g., Welsh \& Orosz 2018; Socia et al. 2020) and are therefore amenable to a novel set of experiments and investigations.

Beyond Kepler, the repurposed K2 mission (Howell et al. 2014) also observed transits of a few planets and planet candidates with orbital periods on the order of hundreds of days despite its limited $\sim 75$-day observational baseline between campaigns (Osborn et al. 2016; Vanderburg et al. 2016a; Giles et al. 2018). At even shorter observational baselines still, the ongoing Transiting Exoplanet Survey Satellite (TESS; Ricker et al. 2015) mission is contributing to the set of long-period exoplanets through single-transit (or monotransit) events (Cooke et al. 2018; Villanueva et al. 2019; Dalba et al. 2020b; Díaz et al. 2020; Eisner et al. 2020; Gill et al. 2020; Lendl et al. 2020). However, during TESS's primary mission, small patches of the sky (near the ecliptic poles) received nearcontinuous observations for almost a year. This strategy allows for the detection of two consecutive transits of an exoplanet with an orbital period on the order of 100 days. Moreover, TESS will observe many single-transit planet candidate host stars again during its extended mission and may detect additional transits that refine the ephemerides (e.g., Cooke et al. 2021).

Only a fraction of the exoplanets discovered in transit surveys are subject to follow-up mass measurement through RV monitoring. Stellar activity, rotational velocity, and the amplitude of RV variations induced by the planet relative to the precision of the facility are all factors that reduce the number of systems amenable to this characterization technique. The latter effect is crucial for long-period exoplanets, as the RV semiamplitude scales inversely with orbital period. There is also the issue that acquiring RV phase coverage for longer-period planets takes more time and requires longer-term stability of the facility. Yet planetary confirmation through mass measurement is especially critical for giant planet candidates with $P \gtrsim 100$ days that have been found to have a false-positive rate greater than $50 \%$ in transit surveys (Santerne et al. 2016). However, since long-period orbits require long-duration follow-up campaigns, the number of longperiod exoplanets with precise mass and radius is further limited (e.g., Dubber et al. 2019).

Here, we add a new member to the sample of exoplanets with $P>100$ days and precisely measured radii and masses: Kepler-1514 b (KOI-3681.01, KIC 2581316). Kepler-1514 b is a statistically validated, Jupiter-size planet (Morton et al. 2016) that was found to have variations in the timing, depth, and duration of its transits (Holczer et al. 2016). The Kepler-1514 system also contains a Kepler Object of Interest (KOI) planet candidate, KOI-3681.02, with a shallower transit and a 10.5-day orbital period, which we validate as Kepler-1514 c. Kepler-1514 therefore joins the list of systems with interior Earth-sized or super-Earth-sized exoplanets with exterior giant planet companions (e.g., Zhu \& Wu 2018; Bryan et al. 2019). The host star itself has a $V$-band magnitude of 11.8 , which is brighter than $96 \%$ of other stars with planets on long-period $(P>100$ days) orbits discovered by Kepler.

The rest of this paper is organized as follows. In Section 2, we describe the photometry of the Kepler-1514 system from the primary Kepler mission and our spectroscopic follow-up observations from the Keck I telescope. In Section 3, we conduct a global modeling of the photometric and spectroscopic data to infer the various stellar, planetary, and orbital properties of the objects in the Kepler-1514 system. Also, we tailor our approach to investigate how the observed rotational variability of Kepler-1514 affects the inferred transit properties of Kepler-1514 b. In Section 4.1, we confirm the planetary nature of Kepler-1514 b by measuring its mass, and we statistically validate KOI-3681.02. In Section 5, we discuss the properties Kepler-1514 b and its host star relative to the sample of other weakly irradiated, cool giant exoplanets. Finally, in Section 6, we summarize our findings.

\section{Observations}

We employ photometric, spectroscopic, and imaging observations in this analysis of the Kepler-1514 system. In the following sections, we describe how each of these data sets was collected and processed.

\subsection{Photometric Data from Kepler}

The Kepler spacecraft observed Kepler-1514 in 18 quarters of its primary mission. These observations captured seven transits of the outer planet Kepler-1514 b and over 100 transits of the inner planet candidate KOI-3681.02. We accessed the simple aperture photometry (SAP) and pre-search data conditioning (PDC) light curves (Jenkins et al. 2010; Smith et al. 2012; Stumpe et al. 2012) from Kepler through the Milkuski Archive for Space Telescopes (MAST). Both types of photometry contain significant brightness variations. The SAP light curves contain systematic variations induced by spacecraft motion and stellar variability, while the PDC light curves contain variations introduced by the detrending. In either case, special consideration is required to model the transit events. We proceed with the SAP data products to ensure that the PDC systematics correction does not distort the deep, long-duration transits of Kepler-1514 b. The crowding metric for each quarter is $\sim 1$, suggesting that the Kepler photometric apertures and resulting radius measurements are not contaminated by background sources (also see Section 2.3). We also verify that the apertures are not contaminated by so-called "phantom stars," which are nonexistent sources often resulting from errors in allsky photometric catalogs (Dalba et al. 2017).

In Figure 1, we show the Quarter 9 transit of Kepler-1514 b to illustrate the typical level of variability present in the SAP and PDC light curves. A previous analysis of the Kepler PDC photometry of Kepler-1514 measured variations in transit timing (TTV), depth (T $\delta \mathrm{V})$, and duration (TDV) for Kepler$1514 \mathrm{~b}$, although the statistical significance of these measurements was low (Holczer et al. 2016). Stellar variability, including brightness variations caused by spots, can cause transit ephemeris variations (e.g., Alonso et al. 2008; Oshagh et al. 2013). Holczer et al. (2016) employed a photometric 

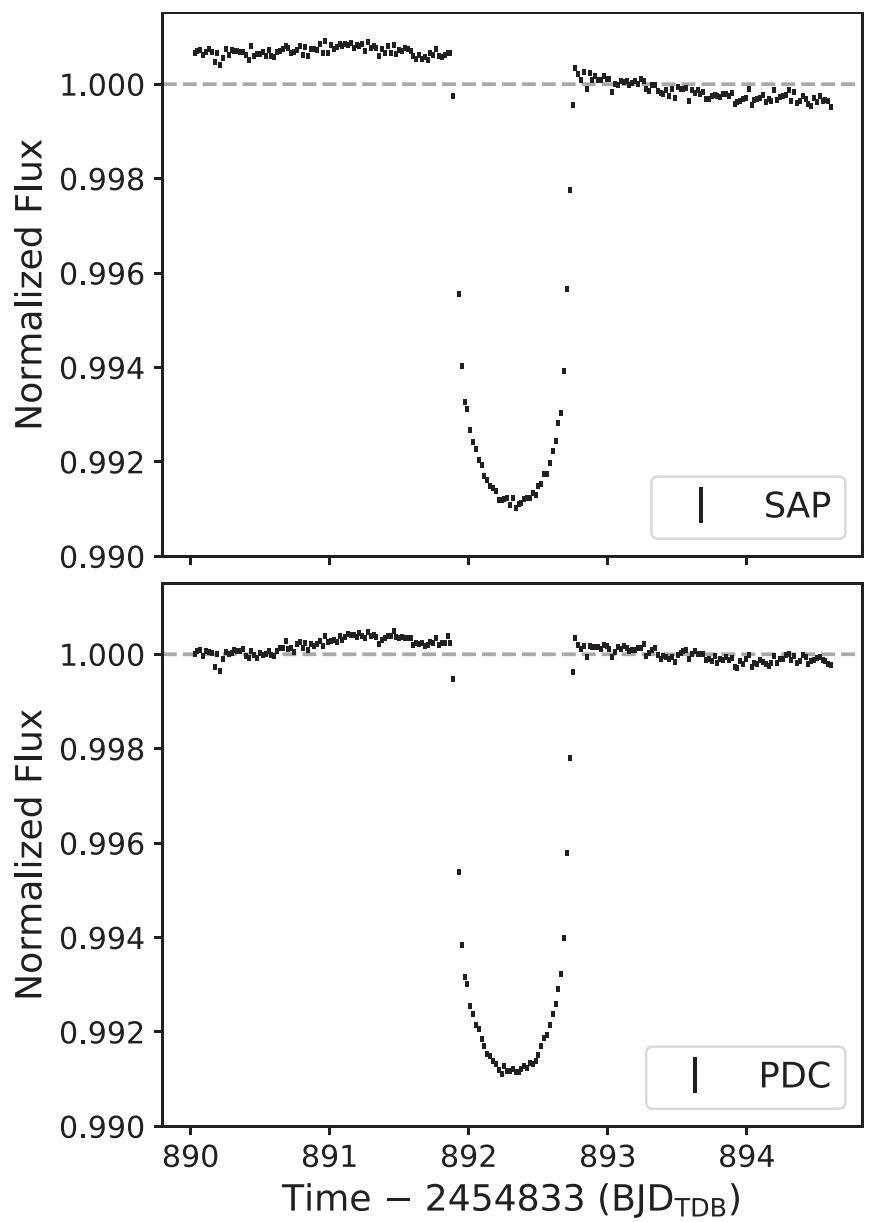

Figure 1. Median-normalized, transit light curve of Kepler-1514 b from Quarter 9 using Kepler SAP (top) and PDC (bottom) data products. We explore whether the variability that is present in these light curves could account for the TTVs, T $\delta$ Vs, and TDVs measured by Holczer et al. (2016) in our modeling of this system.

detrending algorithm to prevent the false detection of TTVs due to stellar variability, but their efforts were spread across a wide catalog of stars and transiting planets. The low statistical significance of the purported transit variations, combined with the variability present in the Kepler light curves of Kepler1514, warrants the focused detrending procedures that we employ in Section 3.

\subsection{Spectroscopic Data from HIRES}

We acquired 12 high-resolution spectra of Kepler-1514 with the High Resolution Echelle Spectrometer (HIRES; Vogt et al. 1994) on the Keck I telescope. One spectrum was acquired with a high signal-to-noise ratio $(\mathrm{S} / \mathrm{N})$ of $\sim 190$ without a heated iodine in the light path. This spectrum is used for a spectroscopic analysis of Kepler-1514 and is vetted for a second set of spectral lines following the methods of Kolbl et al. (2015). We rule out additional spectral lines brighter than $1 \%$ of the primary's and at velocity separations greater than $10 \mathrm{~km} \mathrm{~s}^{-1}$. This high-S/N spectrum also served as a spectral template in the standard forward modeling procedures employed by the California Planet Search (e.g., Howard et al. 2010; Howard \& Fulton 2016), thereby removing the need to synthesize a spectral template (Fulton et al. 2015) or match Kepler-1514 to another star in the HIRES template library (Dalba et al. 2020a). The RVs are listed
Table 1

RV Measurements of Kepler-1514

\begin{tabular}{lcc}
\hline \hline $\mathrm{BJD}_{\mathrm{TDB}}$ & $\mathrm{RV}\left(\mathrm{m} \mathrm{s}^{-1}\right)$ & $S_{\mathrm{HK}}$ \\
\hline $2,458,346.85153$ & $40.6 \pm 4.3$ & $0.139 \pm 0.001$ \\
$2,458,361.02310$ & $12.5 \pm 4.4$ & $0.141 \pm 0.001$ \\
$2,458,390.72137$ & $-56.7 \pm 3.9$ & $0.140 \pm 0.001$ \\
$2,458,396.76976$ & $-68.6 \pm 5.0$ & $0.140 \pm 0.001$ \\
$2,458,560.14495$ & $39.2 \pm 4.2$ & $0.135 \pm 0.001$ \\
$2,458,622.94024$ & $-85.3 \pm 3.8$ & $0.145 \pm 0.001$ \\
$2,458,650.97962$ & $-113.1 \pm 4.0$ & $0.146 \pm 0.001$ \\
$2,458,663.07909$ & $-97.8 \pm 4.2$ & $0.142 \pm 0.001$ \\
$2,458,737.82511$ & $158.4 \pm 4.3$ & $0.131 \pm 0.001$ \\
$2,458,787.84946$ & $25.3 \pm 3.8$ & $0.135 \pm 0.001$ \\
$2,458,906.15457$ & $141.3 \pm 3.8$ & $0.128 \pm 0.001$ \\
\hline
\end{tabular}

in Table 1. Since the HIRES spectra include the Ca II H and K spectral lines, each value of RV is accompanied by a correspond $S_{\mathrm{HK}}$ activity indicator (Isaacson \& Fischer 2010).

\subsection{Archival Imaging Data from NIRC2}

Kepler-1514 was observed at high angular resolution by Kraus et al. (2016) on 2014 August 12 using the NIRC2 adaptive optics imager at Keck Observatory (Wizinowich et al. 2000). The observation used adaptive optics imaging, coronagraphy, and nonredundant aperture mask interferometry to reveal a neighbor located $\rho=0$ ". 272 away from the apparent planethosting star with an apparent contrast of $\Delta K^{\prime}=6.06$ mag, while also achieving deep and close limits for any additional neighbors that might account for the transit signals. This system was also observed with speckle imaging at visible wavelengths at the Wisconsin-Indiana-Yale-NOAO (WIYN) telescope using the DSSI speckle camera (Furlan et al. 2017). The neighbor was not detected, but at 0.27 projected separation the speckle observations yielded relative contrast limits of $\Delta m_{692}=3.05$ mag and $\Delta m_{880}=2.50$ mag.

Kepler-1514 was also observed with Keck II/NIRC2 on 2013 July 7 (as reported by Furlan et al. 2017) and on 2015 July 26 (PI Dupuy). The proper motion of Kepler-1514 is

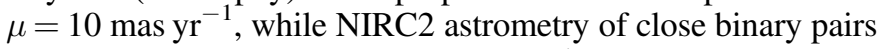
can be measured with a precision of $\lesssim 1-2$ mas (e.g., Dupuy et al. 2016), so the $2 \mathrm{yr}$ baseline offers the opportunity to distinguish whether the neighbor is a comoving low-mass companion or a chance alignment with a background star. We therefore have analyzed the images from all three epochs using the same methods described in Kraus et al. (2016). To briefly recap, our pipeline fits each image of the close pair with a double point-spread function (PSF) model based in the bestfitting single-star PSF selected from all those observed nearby in time, and then the relative astrometry is corrected for the known optical distortion of NIRC2 (Yelda et al. 2010).

In Table 2, we summarize the relative astrometry and photometry that we measured at each epoch, computing a simple mean of the fit results from the individual images. In Figure 2, we plot the corresponding relative motion over time, also showing the trajectories expected for a completely comoving neighbor or a completely nonmoving background star. We find that the background star solution is consistent with the observations $\left(\chi^{2}=8.1\right.$ on 4 degrees of freedom; $P=0.09$ ), whereas the comoving solution is inconsistent with the observations $\left(\chi^{2}=34.6\right.$ on 4 degrees of freedom; $P=5 \times 10^{-7}$ ). The escape velocity of a bound companion at a projected separation of $\rho=0$ ". 272 or 
Table 2

Summary of Kepler-1514 Neighbor Detections from NIRC2 PSF Fitting

\begin{tabular}{|c|c|c|c|c|c|c|}
\hline $\begin{array}{l}\text { Epoch } \\
\text { (MJD) }\end{array}$ & Filter & $N_{\text {obs }}$ & $\begin{array}{c}\rho \\
(\mathrm{mas})\end{array}$ & $\begin{array}{c}P A \\
(\mathrm{deg})\end{array}$ & $\begin{array}{c}\Delta m \\
(\mathrm{mag})\end{array}$ & PI \\
\hline $56,480.53$ & $\mathrm{Brg}$ & 11 & $266.68 \pm 2.05$ & $285.482 \pm 1.328$ & $6.130 \pm 0.164$ & Weaver \\
\hline $56,881.51$ & $\mathrm{Kp}$ & 2 & $270.03 \pm 1.75$ & $284.517 \pm 0.313$ & $6.062 \pm 0.033$ & Kraus \\
\hline $57,229.56$ & $\mathrm{Kp}$ & 6 & $279.41 \pm 1.57$ & $283.609 \pm 0.296$ & $6.240 \pm 0.060$ & Dupuy \\
\hline
\end{tabular}
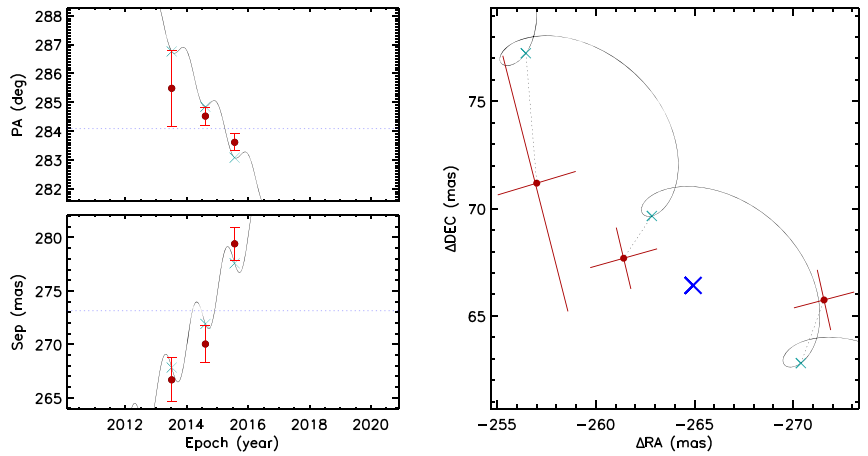

Figure 2. Relative motion of the close neighbor to Kepler-1514, as measured from multiepoch astrometry using adaptive optics imaging. The left panels show the separation and position angle between Kepler-1514 and its neighbor as a function of time, while the right panel shows the relative motion of the neighbor in the plane of the sky. The expected trajectory of a nonmoving background star is shown with the solid curve, while the expected relative position of a comoving binary companion is shown with dotted lines in the left panels and a blue cross in the right panel. We conclude that the faint neighbor is not bound to Kepler-1514 and is instead a chance alignment with an unrelated field interloper.

$\rho=110 \mathrm{au}$ would only be $\Delta v_{\mathrm{esc}} \sim 3 \mathrm{~km} \mathrm{~s}^{-1}$ or $\Delta \mu_{\mathrm{esc}} \sim 1.5$ mas $\mathrm{yr}^{-1}$, much lower than the measured relative motion. We therefore conclude that the relative motion cannot be orbital motion and that the neighbor is a field star seen in chance alignment, not a bound binary companion.

Distant background stars are likely to be relatively blue early-type dwarfs, so the contrast in the Kepler bandpass is likely to be similar to that in the near-infrared $\left(\Delta K^{\prime}=6 \mathrm{mag}\right)$. Under this assumption, the transit depth is only diluted by $0.4 \%$, leading to a planet radius change of $0.2 \%$, well within the measured uncertainty. Therefore, we hereafter neglect any flux contribution that this neighbor made in the transit fits, and we show in Section 4.2 that the signal from KOI-3681.02 cannot originate from this faint field interloper.

\section{Modeling Stellar and Planetary Parameters}

We conducted joint modeling of the stellar, transit, and RV data of Kepler-1514 to infer various stellar, planetary, and systemic parameters using the EXOFASTv2 modeling suite (Eastman et al. 2013; Eastman 2017; Eastman et al. 2019). Since the photometric variability tied to the rotation of Kepler1514 can affect the derived transit parameters, we first applied special detrending to remove this rotational modulation. Then, we conducted an initial EXOFASTv2 fit to assess the impact of this detrending on the variations in transit parameters previously measured for Kepler-1514b. Finally, we ran a comprehensive EXOFASTv2 fit that models the Kepler$1514 \mathrm{~b}$ and the KOI-3681.02 from which we derive the final system parameters.

\subsection{Removal of Out-of-transit Photometric Variability}

The SAP light curves contain long-term variations due to stellar activity and instrumental drifts. These are dominated by differential velocity aberration (DVA), which is the change in the local pixel scale and distortion of the scene caused by spacecraft motion (e.g., Kinemuchi et al. 2012). DVA yields a linear or quadratic slope over the duration of a Kepler quarter that is negligible on the $21 \mathrm{hr}$ timescale of transit. We modeled these variations with a basis spline that we fit simultaneously with the shape of the two transit signals for Kepler-1514 b and KOI-3681.02. Our strategy is similar to that of Vanderburg et al. (2016b), except that we do not also model spacecraft systematic noise in our well-behaved Kepler data. ${ }^{15}$ In brief, we started by clipping anomalous data taken during the following time intervals (given in BKJD, or BJD -2,454,833): $247<t<260,1160.5<t<1162$, and $1289<t<1296$. We identified all gaps in the light curve longer than 0.3 days and introduced discontinuities in our spline at these points. We modeled the two transit signals with analytic Mandel \& Agol (2002) curves and minimized $\chi^{2}$ with a Levenberg-Marquardt algorithm (Markwardt 2009). At each step of the minimization, we calculated the transit models, subtracted them from the light curve, and then fit the basis spline to this residual curve. We then minimized the deviations of (data - transit model spline). After the optimization concluded, we calculated a final spline from the residuals to the best-fit transit model and subtracted it from the light curve to remove the long-term variability.

\subsection{Preliminary EXOFASTv2 Modeling}

After detrending the light curves of Kepler-1514, we completed a preliminary model fit to the transit and RV data using EXOFASTv2. The purpose of this fit was to determine whether the detrending affected the TTVs and T $\delta$ Vs measured previously by Holczer et al. (2016), so we allowed extra parameters describing the timing and depth of each transit. We did not investigate TDVs, as the values measured by Holczer et al. (2016) are fully consistent with no variation in transit duration. We only included transits of Kepler-1514 b in the fit. The fit converged according to the default EXOFASTv2 statistics for each parameter: the number of independent draws of the underlying posterior probability distribution $\left(T_{z}>1000\right.$; Ford 2006) and the well-known Gelman-Rubin statistic $(\mathrm{GR}<1.01$; Gelman \& Rubin 1992).

We show the values of TTVs and T $\delta$ Vs inferred from this preliminary modeling along with those values from Holczer et al. (2016) in Figure 3. The TTVs are presented as the difference between the observed ephemeris and the calculated (linear) ephemeris (i.e., $\mathrm{O}-\mathrm{C}$ ). The $\mathrm{T} \delta \mathrm{Vs}$ were fit relative to the first transit but are shown as median-subtracted values in

\footnotetext{
15 https://github.com/avanderburg/keplerspline.
} 


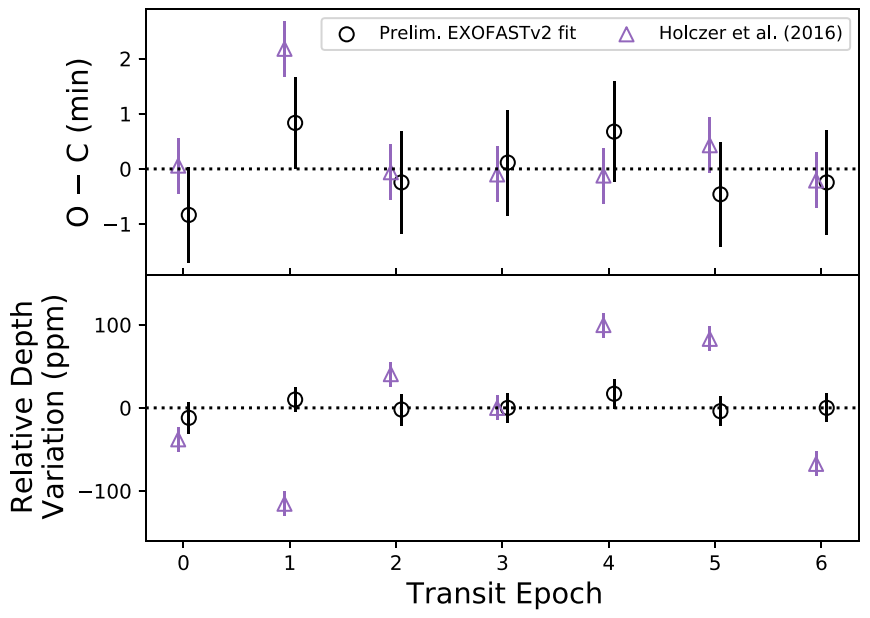

Figure 3. Observed minus calculated $(\mathrm{O}-\mathrm{C})$ timing of the transits (top) and transit depth variations fit relative to the first transit and then median-subtracted (bottom) of Kepler-1514 b from the preliminary EXOFASTv2 fit (Section 3.2). The data sets have been offset horizontally for clarity. In both panels, corresponding values from Holczer et al. (2016) are shown. When detrending the light curves with a spline, we find that the transit depth variations become insignificant.

Figure 3. The TTVs we measure are consistent with, although slightly less precise than, those reported by Holczer et al. (2016). We quantify their significance as the reduced $\chi^{2}$ statistic when compared to a linear ephemeris (i.e., a flat line at $\mathrm{O}-\mathrm{C}=0$ ), which equals 0.5 . Although weak, we cannot claim that these TTVs are negligible, nor can we distinguish between photometric variability and dynamical interaction as their cause. Consequently, we decide to include TTVs in the comprehensive modeling of the Kepler-1514 system data.

On the other hand, we do not detect $\mathrm{T} \delta \mathrm{Vs}$ in the Kepler$1514 \mathrm{~b}$ transits, a result that is inconsistent with Holczer et al. (2016). This discrepancy suggests photometric detrending as the probable cause of the purported T $\delta$ Vs. On this basis, we do not include T $\delta$ Vs in the modeling of the Kepler-1514 system hereafter.

\subsection{Final, Comprehensive EXOFASTv2 Modeling}

For the final global analysis presented in Tables 3 and 4, we conduct the EXOFASTV2 fit in the following fashion. We jointly fit the available detrended Kepler light curve for both planets, but we only fit the Keck-HIRES RVs and allow for TTVs for Kepler-1514b. We exclude fitting the RVs for KOI3681.02 since the measured size from our fit $\left(1.15 R_{\oplus}\right)$ suggests a planet mass on the order of $\sim 1 M_{\oplus}$. A $1 M_{\oplus}$ planet on a circular orbit would produce an RV semiamplitude of $\sim 26 \mathrm{~cm} \mathrm{~s}^{-1}$, which is below the internal precision of the Keck-HIRES measurements and may not be detectable with any amount of data. Within the fit, the host star parameters were determined using the spectral energy distribution (SED) from broadband photometry and the MESA Isochrones and Stellar Tracks (MIST) stellar evolution models (Paxton et al. 2011, 2013, 2015; Choi et al. 2016; Dotter 2016). We place a Gaussian prior of $2.5705 \pm 0.0418$ mas on parallax based on measurements from Gaia (Gaia Collaboration et al. 2018), which we correct for the offset reported by Stassun \& Torres (2018). We also place a Gaussian prior on the stellar metallicity $([\mathrm{Fe} / \mathrm{H}]=$ $0.05 \pm 0.09$ ) based on spectroscopic analysis of the high-S/N template spectrum following Yee et al. (2017). Lastly, we employ an upper limit on the line-of-sight extinction $\left(\mathrm{A}_{V}<0.5115\right)$ from
Table 3

Median Values and 68\% Confidence Intervals for Kepler-1514 Stellar Parameters

\begin{tabular}{|c|c|c|}
\hline Parameter & Units & Values \\
\hline \multicolumn{3}{|c|}{ Informative priors: } \\
\hline$[\mathrm{Fe} / \mathrm{H}]$ & Metallicity (dex) & $\mathcal{N}(0.05,0.09)$ \\
\hline$\varpi$ & Parallax (mas) & $\mathcal{N}(2.5705,0.0418)$ \\
\hline$A_{V}$ & $V$-band extinction (mag) & $\mathcal{U}(0,0.5115)$ \\
\hline \multicolumn{3}{|c|}{ Stellar parameters: } \\
\hline$M_{*}$ & Mass $\left(M_{\odot}\right)$ & $1.196_{-0.063}^{+0.065}$ \\
\hline$R_{*}$ & Radius $\left(R_{\odot}\right)$ & $1.289_{-0.026}^{+0.027}$ \\
\hline$L_{*}$ & Luminosity $\left(L_{\odot}\right)$ & $2.13_{-0.12}^{+0.16}$ \\
\hline$F_{\text {Bol }}$ & Bolometric flux (cgs) & $4.49 \times 10^{-10_{-2.0 \times 10^{-11}}^{+3.1 \times 10^{-11}}}$ \\
\hline$\rho_{*}$ & Density $\left(\mathrm{g} \mathrm{cm}^{-3}\right)$ & $0.787_{-0.040}^{+0.041}$ \\
\hline $\log g$ & Surface gravity (cgs) & $4.295 \pm 0.019$ \\
\hline$T_{\text {eff }}$ & Effective temperature $(\mathrm{K})$ & $6145_{-80}^{+99}$ \\
\hline$[\mathrm{Fe} / \mathrm{H}]$ & Metallicity (dex) & $0.119_{-0.075}^{+0.080}$ \\
\hline$[\mathrm{Fe} / \mathrm{H}]_{0}$ & Initial metallicity $^{\mathrm{a}}$ & $0.163_{-0.064}^{+0.066}$ \\
\hline Age & Age (Gyr) & $2.9_{-1.3}^{+1.6}$ \\
\hline EEP & Equal evolutionary phase $^{\mathrm{b}}$ & $361_{-24}^{+34}$ \\
\hline$A_{V}$ & $V$-band extinction (mag) & $0.076_{-0.053}^{+0.077}$ \\
\hline$\sigma_{\mathrm{SED}}$ & SED photometry error scaling & $0.70_{-0.16}^{+0.25}$ \\
\hline$\varpi$ & Parallax (mas) & $2.568 \pm 0.040$ \\
\hline$d$ & Distance (pc) & $389.3_{-5.9}^{+6.1}$ \\
\hline \multicolumn{2}{|c|}{ Wavelength parameters: } & Kepler \\
\hline$u_{1}$ & Linear limb-darkening coeff. & $0.3474_{-0.0077}^{+0.0076}$ \\
\hline$u_{2}$ & Quadratic limb-darkening coeff. & $0.248 \pm 0.016$ \\
\hline
\end{tabular}

Notes. See Table 3 in Eastman et al. (2019) for a detailed description of all parameters and all default (noninformative) priors beyond those specified here.

${ }^{a}$ Initial metallicity is that of the star when it formed.

${ }^{\mathrm{b}}$ Corresponds to static points in a star's evolutionary history. See Section 2 in Dotter (2016).

the Schlegel et al. (1998) galactic dust maps. We allow the fit to proceed until convergence as quantified by at least 1000 independent draws from the posterior probability distribution of each fitted parameter (Ford 2006) and by a Gelman-Rubin statistic of less than or equal to 1.01 for each fitted parameter (Gelman \& Rubin 1992). The stellar and planetary parameters inferred from the comprehensive EXOFASTV2 modeling are listed in Table 3 and 4, respectively. The final transit and RV data sets along with the best-fit models for the Kepler-1514 system are presented in Figures 4, 5, and 6.

The final TTVs for Kepler-1514 b are shown (as O-C values) in Figure 7. As in the preliminary EXOFASTv2 modeling, the statistical significance of the TTVs is weak. Although we cannot rule out dynamical interactions with other objects in the Kepler-1514 system as their source, their decreasing significance when incorporated into the system modeling indicates that they are likely the result of detrending and modeling choices related to stellar photometric variability.

\section{Results}

\subsection{Confirming Kepler-1514b}

Kepler-1514 b was originally deemed a planet through statistical validation by Morton et al. (2016). Such validation for transiting exoplanets is fairly common, especially given how readily transiting exoplanets have been discovered. However, at orbital periods up to 400 days, suspected giant planet transit signals have an alarmingly high false-positive 
Table 4

Median Values and 68\% Confidence Interval for the Planets in the Kepler-1514 System

\begin{tabular}{|c|c|c|c|}
\hline \multirow{2}{*}{$\begin{array}{l}\text { Parameter } \\
\text { Planetary parameters: }\end{array}$} & \multirow[t]{2}{*}{ Units } & \multicolumn{2}{|c|}{ Values } \\
\hline & & b & c \\
\hline$P$ & Period (days) & $217.83184 \pm 0.00012$ & $10.514181 \pm 0.000039$ \\
\hline$R_{P}$ & Radius $\left(R_{\mathrm{J}}\right)$ & $1.108 \pm 0.023$ & $0.1049_{-0.0039}^{+0.0051}$ \\
\hline$M_{P}$ & Mass $\left(M_{\mathrm{J}}\right)$ & $5.28 \pm 0.22$ & $\cdots$ \\
\hline$T_{C}$ & Time of conjunction ${ }^{\mathrm{a}}\left(\mathrm{BJD}_{\mathrm{TDB}}\right)$ & $2,455,071.81411 \pm 0.00046$ & $2,454,957.0546_{-0.0036}^{+0.003}$ \\
\hline$a$ & Semimajor axis (AU) & $0.753_{-0.014}^{+0.013}$ & $0.0997 \pm 0.0018$ \\
\hline$i$ & Inclination (deg) & $89.944_{-0.010}^{+0.013}$ & $87.98_{-0.40}^{+1.2}$ \\
\hline$e$ & Eccentricity $^{\mathrm{b}}$ & $0.401_{-0.014}^{+0.013}$ & $0.32_{-0.19}^{+0.35}$ \\
\hline$\omega_{*}$ & Argument of periastron (deg) & $-75.28_{-0.71}^{+0.75}$ & $0_{-160}^{+120}$ \\
\hline$T_{\text {eq }}$ & Equilibrium temperature $^{\mathrm{c}}(\mathrm{K})$ & $387.9_{-5.0}^{+6.0}$ & $1066_{-14}^{+16}$ \\
\hline$K$ & RV semiamplitude $\left(\mathrm{m} \mathrm{s}^{-1}\right)$ & $172.5 \pm 3.9$ & $\ldots$ \\
\hline$R_{P} / R_{*}$ & Radius of planet in stellar radii & $0.08835_{-0.00015}^{+0.00014}$ & $0.00836_{-0.00026}^{+0.00037}$ \\
\hline$a / R_{*}$ & Semimajor axis in stellar radii & $125.6 \pm 2.2$ & $16.63 \pm 0.29$ \\
\hline$\delta$ & Transit depth (fraction) & $0.007805_{-0.000027}^{+0.000025}$ & $0.0000699_{-0.0000043}^{+0.0000063}$ \\
\hline$\tau$ & Ingress/egress transit duration (days) & $0.07409_{-0.00097}^{+0.00093}$ & $0.00166_{-0.00036}^{+0.00091}$ \\
\hline$T_{14}$ & Total transit duration (days) & $0.88862_{-0.00078}^{+0.00077}$ & $0.1567_{-0.0034}^{+0.0035}$ \\
\hline$b$ & Transit impact parameter & $0.169_{-0.039}^{+0.030}$ & $0.47_{-0.31}^{+0.22}$ \\
\hline$b_{S}$ & Eclipse impact parameter & $0.074_{-0.017}^{+0.013}$ & $0.44_{-0.28}^{+0.18}$ \\
\hline$\tau_{S}$ & Ingress/egress eclipse duration (days) & $0.0323 \pm 0.0011$ & $0.00195_{-0.00083}^{+0.00035}$ \\
\hline$T_{S, 14}$ & Total eclipse duration (days) & $0.395 \pm 0.013$ & $0.160_{-0.042}^{+0.058}$ \\
\hline$\rho_{P}$ & Density $\left(\mathrm{g} \mathrm{cm}^{-3}\right)$ & $4.82_{-0.25}^{+0.26}$ & $\ldots$ \\
\hline $\log g_{P}$ & Surface gravity & $4.028 \pm 0.017$ & $\cdots$ \\
\hline$\langle\ldots\rangle$ & Incident flux $\left(10^{9} \mathrm{erg} \mathrm{s}^{-1} \mathrm{~cm}^{-2}\right)$ & $0.00440_{-0.00024}^{+0.00029}$ & $0.263_{-0.066}^{+0.029}$ \\
\hline$T_{P}$ & Time of periastron $\left(\mathrm{BJD}_{\mathrm{TDB}}\right)$ & $2,454,981.75_{-0.74}^{+0.73}$ & $2,454,956.9 \pm 1.3$ \\
\hline$T_{S}$ & Time of eclipse (BJD $\left.\mathrm{TDB}_{\mathrm{TD}}\right)$ & $2,455,196.06_{-0.63}^{+0.61}$ & $2,454,951.8_{-2.9}^{+2.7}$ \\
\hline$T_{A}$ & Time of ascending node $\left(\mathrm{BJD}_{\mathrm{TDB}}\right)$ & $2,455,002.91_{-0.78}^{+0.82}$ & $2,454,954.8_{-1.5}^{+1.3}$ \\
\hline$T_{D}$ & Time of descending node $\left(\mathrm{BJD}_{\mathrm{TDB}}\right)$ & $2,455,164.5 \pm 1.2$ & $2,454,959.3_{-1.3}^{+1.5}$ \\
\hline$e \cos \omega_{*}$ & & $0.1021_{-0.0041}^{+0.0040}$ & $0.00 \pm 0.42$ \\
\hline$e \sin \omega_{*}$ & & $-0.388 \pm 0.014$ & $0.02_{-0.27}^{+0.17}$ \\
\hline$P_{S}$ & A priori nongrazing eclipse prob. & $0.01201 \pm 0.00011$ & $0.063_{-0.011}^{+0.065}$ \\
\hline$P_{S, G}$ & A priori eclipse prob. & $0.01434 \pm 0.00013$ & $0.064_{-0.011}^{+0.066}$ \\
\hline Telescope parameters: & & Keck I & \\
\hline$\gamma_{\text {rel }}$ & Relative RV offset $\left(\mathrm{m} \mathrm{s}^{-1}\right)$ & $38.9 \pm 2.1$ & \\
\hline$\sigma_{J}$ & $\mathrm{RV}$ jitter $\left(\mathrm{m} \mathrm{s}^{-1}\right)$ & $4.2_{-2.8}^{+3.2}$ & \\
\hline$\sigma_{J}^{2}$ & $\mathrm{RV}$ jitter variance & $17_{-16}^{+38}$ & \\
\hline
\end{tabular}

Notes. See Table 3 in Eastman et al. (2019) for a detailed description of all parameters and all default (noninformative) priors.

a Time of conjunction is commonly reported as the "transit time."

${ }^{\mathrm{b}}$ By the Lucy-Sweeney bias (Lucy \& Sweeney 1971), the reported eccentricity of the inner planet (Kepler-1514c) is not significant. The orbit should be interpreted as consistent with circular.

${ }^{\mathrm{c}}$ Assumes no albedo and perfect redistribution.

probability (e.g., Santerne et al. 2016). Therefore, mass measurement is needed when confirming the planetary nature of a long-period ( $P \gtrsim 100$ days), giant exoplanet (e.g., Dubber et al. 2019).

We measure the mass of Kepler-1514 b to be $5.28 \pm 0.22 M_{\mathrm{J}}$ and thereby confirm it to be a genuine planet. Its radius is $1.108 \pm 0.023 R_{\mathrm{J}}$, which places its bulk density in the 95th percentile among other weakly irradiated giant exoplanets. It orbits its host star with an orbital period of $217.83184 \pm 0.00012$ days and an orbital eccentricity of $0.401_{-0.014}^{+0.013}$. As we will discuss in the following sections, the combination of stellar, orbital, and planetary properties places it among a small group of interesting and accessible exoplanets.

\subsection{Validating Kepler-1514c}

We did not infer the mass of KOI-3681.02 from the KeckHIRES RVs in the final, comprehensive EXOFASTV2 modeling because its signal is undetectable given the precision of the Keck-HIRES data (Section 3.3). However, we are able to statistically validate the existence of this planet candidate.

We begin by ruling out the possibility of the transit signal originating from the neighbor star detected by Kraus et al. (2016), which we determined is not associated with Kepler1514 (see Section 2.3). We follow the methodology of Vanderburg et al. (2019) to estimate the magnitude difference $(\Delta m)$ between Kepler-1514 and the faintest possible neighbor that could cause the shallow transit signals. Equation (4) of Vanderburg et al. (2019) states

$$
\Delta m \lesssim 2.5 \log _{10}\left(\frac{t_{12}^{2}}{t_{13}^{2} \delta}\right)
$$

where $t_{12}$ is the duration of transit ingress and egress (i.e., from first to second contact), $t_{13}$ is the amount of time between first 

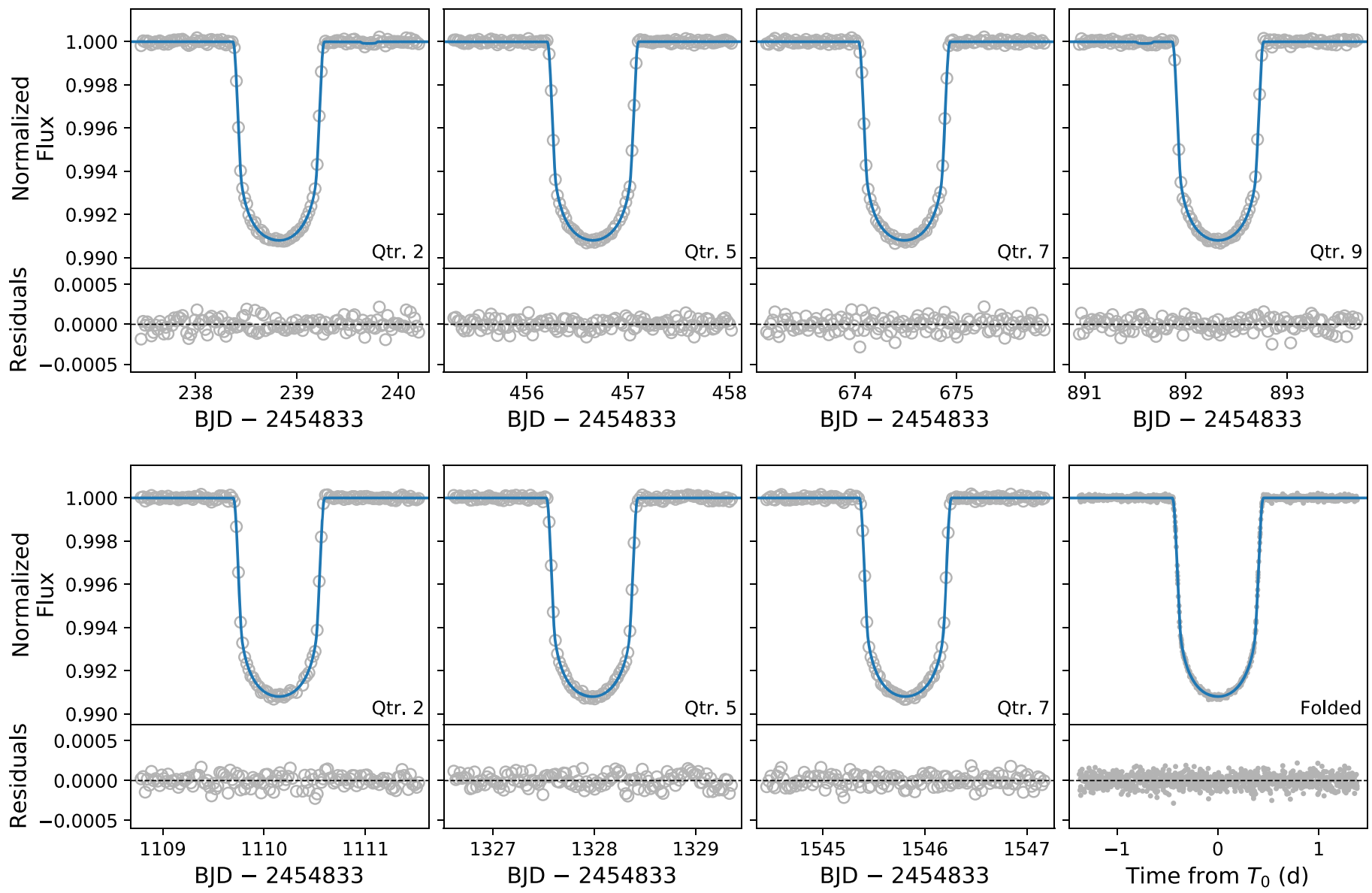

Figure 4. All long-cadence transits of Kepler-1514 b, labeled by Kepler Quarter, and then folded on the best-fit ephemeris in the bottom right panel. The blue lines are

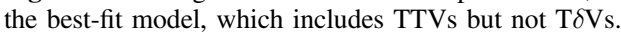

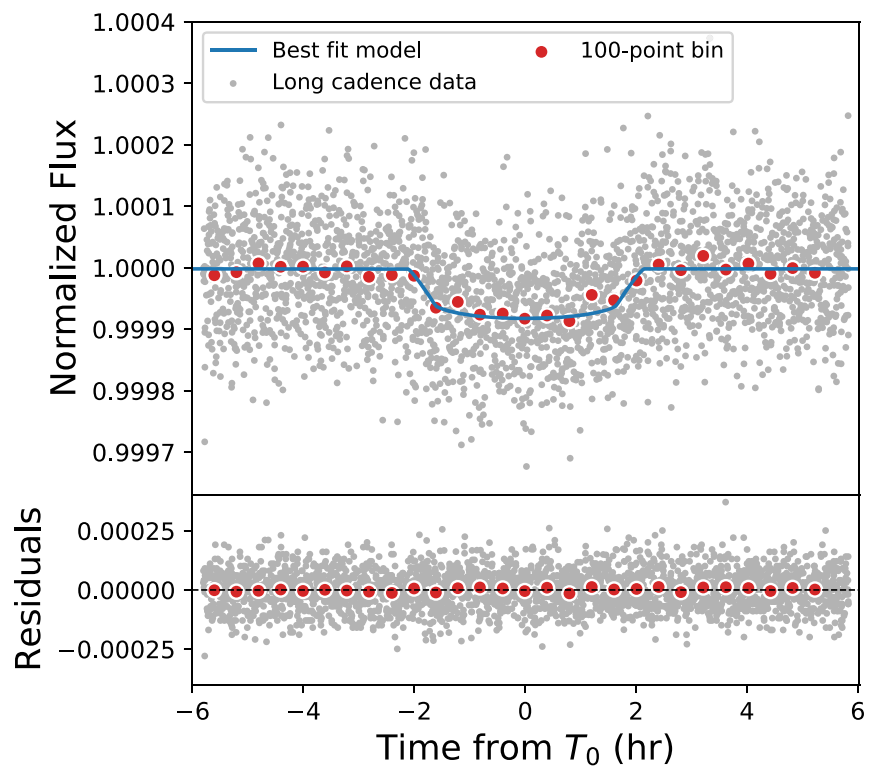

Figure 5. Kepler long-cadence transits of KOI-3681.02 folded on the best-fit ephemeris, which does not include TTVs. The binned data clearly identify the shallow transit of the exoplanet candidate.

and third contact, and $\delta$ is the transit depth. The ingress and egress durations used in this calculation should not be constrained by stellar density, so we do not use results of the stellar modeling from Section 3. Instead, we conduct a new fit to just the transits of KOI-3681.02 using exoplanet ${ }^{16}$ (Foreman-Mackey et al. 2020). This fit does not include any constraints based on stellar properties, and all transit parameters are only bound to physically realistic regions of parameter space. We apply the same convergence criteria for this fit as for the EXOFASTV2 fits described in Section 3. After convergence, we derive values of $t_{12}$ and $t_{13}$ following Equations (14)-(16) of Winn (2010).

From Equation (1), we find the distribution of $\Delta m$ values to be skewed toward zero, with a median of 0.4 mag and a 99th percentile of 3.9 mag. We compare this value to the approximate Kepler-band magnitude of the neighbor star, which we estimate with a stellar population simulation from TRILEGAL (Groenewegen et al. 2002; Girardi et al. 2005; Vanhollebeke et al. 2009) at the equatorial coordinates of Kepler-1514. For simulated stars with $K_{s}$-band magnitudes of $16.7 \pm 0.5$ (i.e., the sum of Kepler-1514's magnitude and the NIRC2 imaging $\Delta m$ ), the distribution of Kepler-band magnitudes has a mean of $19.1 \mathrm{mag}$ and a standard deviation of 0.8 mag. Compared with the Kepler-band magnitude of Kepler1514 (11.69), this yields $\Delta m=7.4 \pm 0.8$. The likely $\Delta m$ of the neighbor star in the Kepler band is $8 \sigma$ discrepant with the median $\Delta m$ calculated in Equation (1) and over $4 \sigma$ discrepant with the 99th percentile of the $\Delta m$ distribution. Therefore, we

$\overline{{ }^{16} \text { https://github.com/exoplanet-dev/exoplanet }}$ 

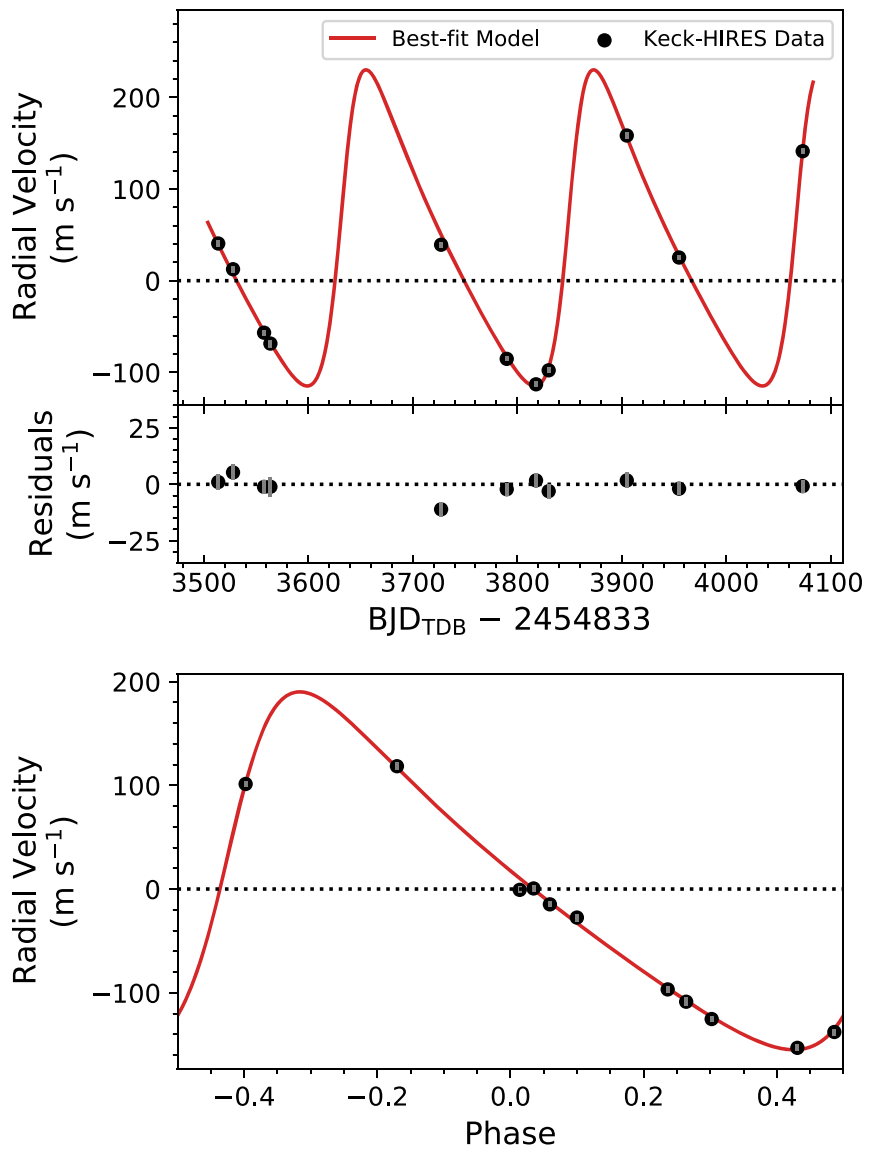

Figure 6. RV measurements of Kepler-1514 from Keck-HIRES. The top panel is the time series data, and the bottom panel shows the data phase folded on the best-fit ephemeris using the time of conjunction $\left(T_{C}\right)$ as the reference point. Error bars are small but are shown in gray in each panel.

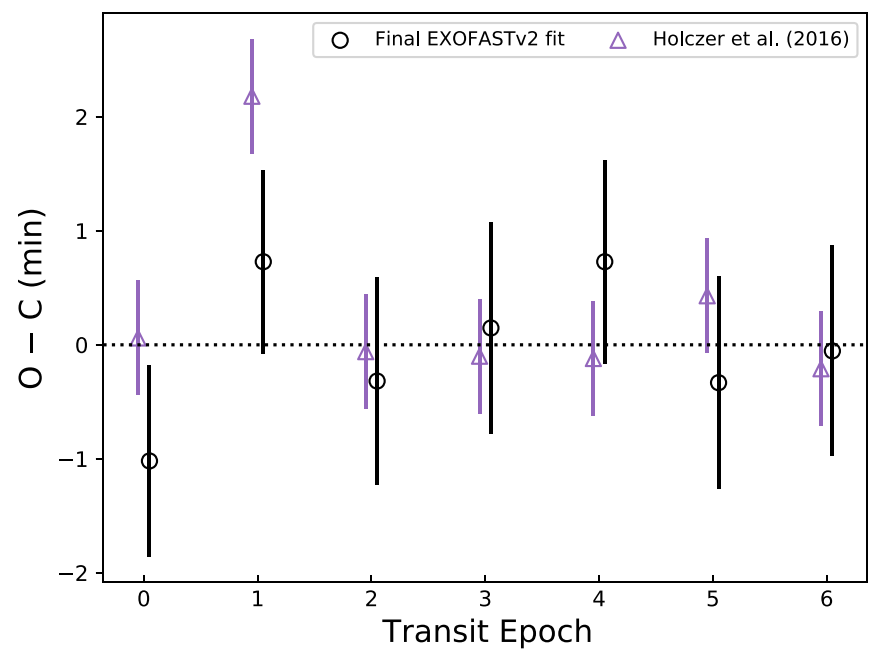

Figure 7. Observed minus calculated $(\mathrm{O}-\mathrm{C})$ timing of the transits of Kepler$1514 \mathrm{~b}$ from the final, comprehensive EXOFASTV2 fit (Section 3.3). The measured times are broadly consistent with a linear ephemeris. The data sets have been offset horizontally for clarity.

confidently rule out the neighbor star at a separation of 0 ". 27 as a possible cause of the KOI-3681.02 transits.

Kraus et al. (2016) also reported the detection of three fainter neighbors $\left(\Delta K^{\prime}=8.4-9.7\right)$ at wider separations 4!' 1-5!"3. The Kepler-band $\Delta m$ values for these stars will be even larger than that of the close neighbor, so we can rule these stars out as the source of the KOI-3681.02 transits by the same argument.

Next, we use VESPA (Morton 2012, 2015) to calculate the false-positive probability of KOI-3681.02. We perform our calculation several times by drawing on the inferred stellar properties and photometry of Kepler-1514 in addition to the contrast curve reported by Kraus et al. (2016). In each calculation, the false-positive probability was below the $1 \%$ threshold typically employed for statistical validation.

The last piece of evidence we provide for the validation of KOI-3681.02 are the results of Lissauer et al. (2012), which show that a vast majority of Kepler multiplanet candidates are indeed genuine planets. Specifically, the study estimates that in systems with one confirmed planet and one planet candidate, the planet candidate is a false positive $<1 \%$ of the time. This combination of this information and that provided above makes a thorough case for the validation of this planet candidate. Therefore, based on our validation analysis, we hereafter refer to KOI-3681.02 as Kepler-1514 c.

\section{Discussion}

\subsection{Tension in Stellar Properties}

The stellar properties of the Kepler-1514 system are constrained by both the SED data and the transit and RV data included in the comprehensive modeling (Section 3.3). We explored how each of these affected the final stellar properties (Table 3) by running two additional EXOFASTv2 fits. The first was a "star only" fit (i.e., with no transit or RV data), and the second was a "no SED" fit (i.e., identical to the global fit but without the SED). In lieu of the SED, we applied a prior to stellar effective temperature $(6073 \pm 110 \mathrm{~K})$ based on spectroscopic analysis of the high-S/N template spectrum. In the "star only" fit, Kepler-1514 was found to be more massive $\left(M_{\star}=1.252_{-0.064}^{+0.050} M_{\odot}\right)$, denser $\left(\rho_{\star}=0.918_{-0.095}^{+0.080} \mathrm{~g} \mathrm{~cm}^{-3}\right)$, and hotter $\left(T_{\text {eff }}=6470 \pm 170 \mathrm{~K}\right)$ when compared to the same parameters in the "no SED" fit $\left(M_{\star}=1.102_{-0.087}^{+0.089} M_{\odot}\right.$, $\left.\rho_{\star}=0.783_{-0.044}^{+0.046} \mathrm{~g} \mathrm{~cm}^{-3}, T_{\text {eff }}=5982_{-87}^{+93} \mathrm{~K}\right)$. The stellar radii inferred from these two fits were consistent, but in mass, density, and effective temperature, the discrepancies were $1.4 \sigma$, $1.3 \sigma$, and $2.5 \sigma$, respectively. Our final solution, as presented in Section 3.3, represents a compromise between these two slightly discrepant solutions, though it is likely that our uncertainties are slightly underestimated. Although this tension is passed down to the planetary parameters as well, it does not affect our interpretation of the planets themselves.

This slight tension is due to a mismatch between the stellar mass and radius from the MIST models and SED, respectively, and the stellar density constrained by the transit duration and eccentricity (Seager \& Mallén-Ornelas 2003). It is unclear which to believe more. On one hand, the transits have a very high $\mathrm{S} / \mathrm{N}$, but half of the RV phase curve is sparsely sampled (i.e., there are only two data points between -0.5 and 0 in Figure 6). If the eccentricity were biased high by either of these points, it would skew the inferred stellar density and could be the source of this tension. While we see no evidence to suggest that either point is problematic, many undetectable problems could lead to significant single-point RV outliers. On the other hand, the stellar models that underlie the MIST and SED constraints have poorly understood systematics. EXOFASTv2 automatically attempts to account for them, but it may not be sufficient. 
One way to further investigate this tension is to acquire more high-precision RV observations that cover the sparsely sampled phases. Ideally, this would eliminate the possibility that the stellar density is being influenced by a single point outlier in the RV data set. The transit and RV data for most exoplanet systems are not precise enough to produce a constraint on stellar density that can overwhelm the stellar information present in the isochrone models and SED, especially when precise Gaia parallax measurements are used. In this way, the Kepler-1514 system could provide valuable future tests of stellar models that otherwise limit measurements of fundamental stellar properties (Tayar et al. 2020).

\subsection{Kepler-1514 b: A Dense, Cool Giant Planet}

When considering Kepler-1514b among other known exoplanets, the foremost point of interest is its transiting geometry despite it 218-day orbit. This property places Kepler$1514 \mathrm{~b}$ in the 98th percentile of transiting exoplanets by orbital period. Considering the planet characterization opportunities enabled by transits, Kepler-1514 b is in an inherently interesting group of exoplanets.

With a longer orbital period also comes a lower stellar irradiation relative to most transiting exoplanets. Kepler-1514 b receives an average incident flux of $4.4 \times 10^{6} \mathrm{erg} \mathrm{s}^{-1} \mathrm{~cm}^{-2}$ (3.2 times that of Earth), which is approximately two orders of magnitude below the empirically determined threshold for radius inflation (Demory \& Seager 2011; Miller \& Fortney 2011). Kepler-1514 b is still informative to investigations of radius inflation, though. Sestovic et al. (2018) found that giant planet radius inflation is a function of planet mass, and for giant planets with $M_{p}>2.5 M_{\mathrm{J}}$, radius inflation is not effective below $\sim 1.6 \times 10^{8} \mathrm{erg} \mathrm{s}^{-1} \mathrm{~cm}^{-2}$ incident flux. However, the weakly irradiated side of this threshold for massive giant planets contains only two planets. Adding Kepler- $1514 \mathrm{~b}$ as a third member to this small group would likely inform the radius inflation boundary for massive planets.

The Jupiter-sized Kepler-1514 b has a bulk density of $4.82_{-0.25}^{+0.26} \mathrm{~g} \mathrm{~cm}^{-3}$, which is consistent with that of other cold, giant planets for which electron degeneracy pressure yields high densities (e.g., Weiss et al. 2013). Among other known giant planets receiving flux below the canonical radius inflation threshold, Kepler-1514 b ranks in the 95th percentile by bulk density (Figure 8, top panel). It marks the upper tail of a distribution of bulk density that spans two orders of magnitude, mirroring a similar spread in planet mass (as indicated by colors of the points in Figure 8).

In mass-radius space (Figure 8, bottom panel), Kepler$1514 \mathrm{~b}$ occupies a region where planet size has become almost entirely independent of mass. Different studies have suggested a range of masses at which electron degeneracy pressure becomes the primary source of support within a giant planet's interior, leading to increasingly more massive objects of nearly the same size. The early theoretical work by Zapolsky \& Salpeter (1969) found this mass to be between 1.2 and $3.3 M_{\mathrm{J}}$ for an isolated sphere of hydrogen and helium. More recent planetary evolution models (Fortney et al. 2007) suggest a range of roughly $2-5 M_{\mathrm{J}}$ depending on composition and stellar irradiation. Empirical measurements of the transition to degenerate cores have included $\sim 0.5 M_{\mathrm{J}}$ (Weiss et al. 2013) and $0.41 \pm 0.07 M_{\mathrm{J}}$ (Chen \& Kipping 2017). The former value was a fiducial boundary that represents a broad peak extending up to several Jupiter masses (see Figure 12 of Weiss et al. 2013),
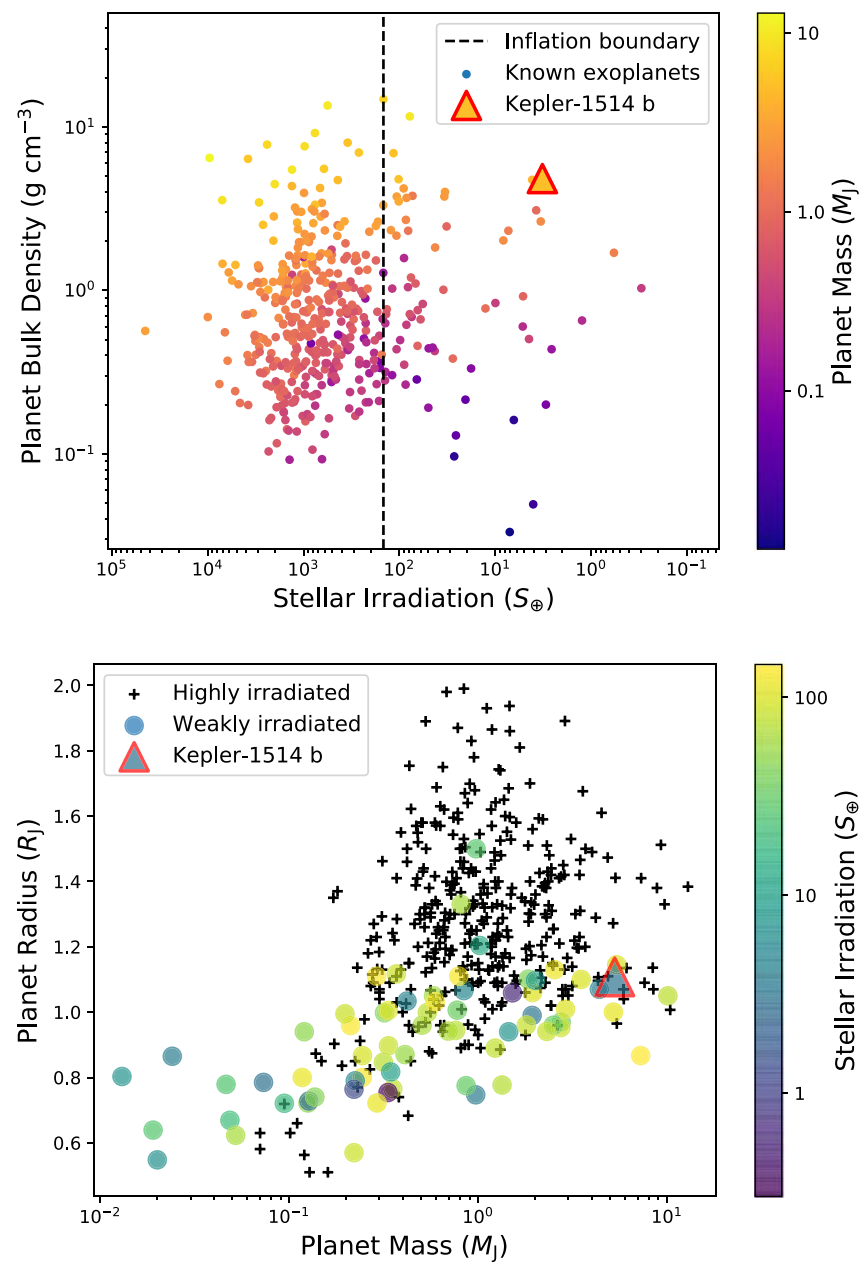

Figure 8. All confirmed giant $\left(R_{p}>0.5 R_{\mathrm{J}}\right)$ exoplanets (from the NASA Exoplanet Archive; accessed 2020 July 9) for which stellar irradiation either was given or could be calculated and planet mass and radius were known to at least $50 \%$ precision. Top: of those planets with stellar irradiation below the empirical inflation boundary (Miller \& Fortney 2011; Demory \& Seager 2011), Kepler-1514 b ranks in the 95th percentile in bulk density. The spread in density is due to the spread in mass, since most of these weakly irradiated giant planets are roughly the same size. Bottom: the inflation boundary from the top panel separates weakly and highly irradiated planets. The combination of high mass and low irradiation for Kepler- $1514 \mathrm{~b}$ places it among a small group of giant planets that are useful for testing models of giant planet interior structure.

while the latter value was inferred from data without assuming prior knowledge of giant planet structure. In either case, the discrepancy with the previously mentioned models may, at least in part, be due to planetary radii that are inflated by physical mechanisms not captured by the models. Nevertheless, at $5.3 M_{\mathrm{J}}$, Kepler-1514 b is likely supported through electron degeneracy pressure. Considering only the weakly irradiated giant planets in Figure 8 (bottom panel), only a few have masses as large as or greater than Kepler-1514b. These planet are valuable laboratories for testing models of giant planet interiors. Kepler-1514 b specifically adds a crucial new data point at high density and low insolation that is especially amenable to explorations of interior metallicity and evolution.

In mass, radius, density, and average stellar irradiation, Kepler- $1514 \mathrm{~b}$ is similar to HD $80606 \mathrm{~b}\left(M_{p} \approx 4.1 M_{\mathrm{J}}, R_{p} \approx 1.0\right.$ $R_{\mathrm{J}}, \rho_{p} \approx 5.1 \mathrm{~g} \mathrm{~cm}^{-3}$, and $S_{p} \approx 4.1 S_{\oplus}$; Bonomo et al. 2017). The orbit of Kepler- $1514 \mathrm{~b}$ is also moderately eccentric, although substantially less than that of HD 80606 b $(e \approx 0.93$; Bonomo et al. 2017). Despite these similarities, their formation histories 
may be different. The high eccentricity of HD $80606 \mathrm{~b}$ is thought to be a remnant of migration driven by an associated stellar companion (e.g., Naef et al. 2001; Moutou et al. 2009). As discussed in Section 2.3, the only known nearby neighbor of Kepler-1514 is a background source. Combined with the semimajor axis and eccentricity of Kepler-1514 b's orbit and the stellar metallicity (i.e., $[\mathrm{Fe} / \mathrm{H}])$, Kepler- $1514 \mathrm{~b}$ may have instead migrated via planet-planet scattering (e.g., Dawson \& Johnson 2018) or within a cavity formed in the protostellar disk, the latter of which is perhaps more consistent with the presence of Kepler-1514 c (Debras et al. 2021). All of the other similarities between Kepler-1514 b and HD 80606 b are interesting to consider in light of possible different migration pathways. Further data, and possibly numerical simulations that include the inner planet Kepler-1514c, would be useful to place stronger constraints on evolutionary theories.

\subsection{Further Study: Interiors, Atmospheres, Obliquity, and Exomoons}

One avenue of continued study is to consider the interior structure of the giant planet Kepler-1514 b. Thorngren et al. (2016) identified a relationship between increasing mass and increasing heavy-element mass for uninflated giant exoplanets. However, for planet mass greater than $\sim 3 M_{\mathrm{J}}$, this relationship was informed by only three data points that showed substantial scatter (see Figure 11 of Thorngren et al. 2016). Furthermore, Thorngren et al. (2016) also identified an inverse relationship between planet mass and metal enrichment relative to stellar for the same sample of weakly irradiated giant planets. As found by the spectroscopic stellar characterization (Section 3.3), Kepler-1514 is only slightly metal-rich $\left([\mathrm{Fe} / \mathrm{H}]=0.119_{-0.075}^{+0.080}\right.$; Table 3). Testing for a weak relative metal enhancement between Kepler-1514 b and its host through a metallicity retrieval or an atmospheric abundance measurement would be helpful to refining both aforementioned relationships.

A key aspect of the amenability of the Kepler-1514 system to the follow-up characterization we have discussed here is the stellar brightness. Kepler-1514 has a $V$-band magnitude of 11.8. Of all the planet host stars discovered by the Kepler primary mission, only 81 are brighter at optical wavelengths. This brightness is especially valuable when comparing to other weakly irradiated giant exoplanet systems with known masses and radii (Figure 9). At similar brightness, only Kepler-16 b receives a lower stellar irradiation. At similar stellar irradiation, only HD $80606 \mathrm{~b}$ is brighter. Together, these three exoplanets are representative of broad diversity in orbital eccentricities of long-period giant planets as well.

Despite the promising brightness of Kepler-1514, prospects for atmospheric characterization via transmission spectroscopy are poor. The high mass of Kepler-1514 b yields a surface gravity of $\sim 107 \mathrm{~m} \mathrm{~s}^{-2}$, much higher than that of Jupiter $\left(\sim 25 \mathrm{~m} \mathrm{~s}^{-2}\right)$ or Saturn $\left(\sim 10 \mathrm{~m} \mathrm{~s}^{-2}\right)$. Adopting the equilibrium temperature (Table 4) and assuming a hydrogen-dominated atmosphere, we estimate an atmospheric scale height of $\sim 15 \mathrm{~km}$. A transmission spectrum feature of a few scale heights would only be $\sim 10$ parts per million, even in the absence of clouds, which is beyond the reach of any current or planned observational facility. Similarly, atmospheric characterization via direct imaging is also challenging, as the separation between Kepler-1514b and its host star is only 2 mas.

Another exciting avenue of further study of Kepler-1514 b is the measurement of stellar obliquity through the Rossiter

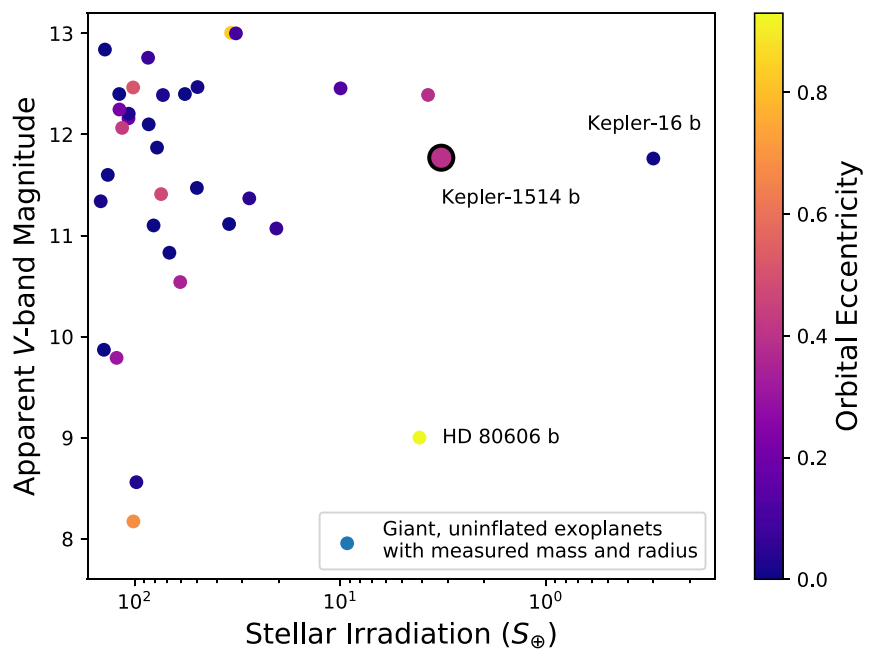

Figure 9. Giant $\left(R_{p}>0.5 R_{\mathrm{J}}\right)$ exoplanets with mass and radius measured to better than $50 \%$ precision that receive stellar irradiation below $2 \times 10^{8} \mathrm{erg} \mathrm{s}^{-1} \mathrm{~cm}^{-2}$ stellar, meaning they are likely uninflated (e.g., Miller \& Fortney 2011; Demory $\&$ Seager 2011; Sestovic et al. 2018). The points are colored by orbital eccentricity.

-McLaughlin (RM) effect (McLaughlin 1924; Rossiter 1924). Spin-orbit alignment plays a key role in planetary migration processes (e.g., Fabrycky \& Tremaine 2007; Chatterjee et al. 2008), so determining this value for Kepler- $1514 \mathrm{~b}$ would be particularly revealing. Using the high-S/N template spectrum of Kepler-1514 acquired with Keck-HIRES (see Section 2.2), we measured the stellar projected rotational velocity $(v \sin i)$ to be $7.8 \pm 1.0 \mathrm{~km} \mathrm{~s}^{-1}$ following the spectral matching technique of Petigura et al. (2017). According to Equation (40) of Winn (2010), we would therefore expect the amplitude of the RM effect to be $\sim 60 \mathrm{~m} \mathrm{~s}^{-1}$. The $21 \mathrm{hr}$ transit duration presents a formidable challenge, though, as it is longer than the maximum length of time that any single site with precise RV capabilities can observe the star. Depending on the transit timing and the precision of the RV facility, it may be possible to detect the RM effect in an observation of a partial transit (i.e., baseline and ingress or egress). The Keck-HIRES observations of Kepler1514 achieved $\sim 5 \mathrm{~m} \mathrm{~s}^{-1}$ internal precision with exposure times between 10 and 19 minutes (depending on observing conditions). Assuming stable 15-minute exposures, we could acquire $\sim 7 \mathrm{RV}$ measurements with $\sim 5 \mathrm{~m} \mathrm{~s}^{-1}$ uncertainty over the $1.78 \mathrm{hr}$ ingress (or egress) with Keck-HIRES. This may be sufficient to constrain the stellar obliquity. Alternatively, the Kepler-1514 system may be an opportunity for a coordinated observing campaign at multiple sites spread out in longitude assuming that the noise properties of both facilities are well characterized. In either case, further effort should be made to explore the extent to which RM measurements of partial transits of long-period exoplanets lead to degeneracies in the solutions for stellar obliquity.

To date, the majority of systems subject to RM measurements host short-period hot Jupiters (see Triaud 2018, for a review). Currently, Kepler-16 is the only system with stellar obliquity measurement from a planet with a longer orbital period $(P=228$ days) than Kepler-1514 b (Winn et al. 2011). However, Kepler-16 is a binary system. This means that Kepler-1514 b is poised to become the longest-period exoplanet with a stellar obliquity measurement in a single star system.

Lastly, we point out the potential of Kepler-1514 b as a host for exomoons. It is plausible that a massive, giant planet with 
an orbital period of several hundred days may harbor a system of exomoons. Teachey et al. (2018) estimated the occurrence of Galilean-size exomoons for exoplanets similar to Kepler$1514 \mathrm{~b}$ to be $0.16_{-0.10}^{+0.13}$. Hill et al. (2018) also discussed the occurrence of exomoons orbiting long-period giant planets discovered by Kepler, suggesting the possible existence of a large population of exomoons within their star's habitable zones. Furthermore, several other efforts to identify exomoons have recognized Kepler-1514 b (Kipping et al. 2012, 2015; Guimarães \& Valio 2018). We demonstrated that Kepler$1514 \mathrm{~b}$ exhibits weak TTVs (Section 3), which could have several explanations, including exomoons (e.g., Sartoretti \& Schneider 1999; Szabó et al. 2006; Simon et al. 2007; Kipping 2009a, 2009b).

However, we currently do not have evidence to support such an extraordinary claim. Relative to the solar system giant planets -which are known to host moons in abundance-Kepler$1514 \mathrm{~b}$ likely experienced a different formation and migration history that may have involved processes that are thought to deplete planets of moons (e.g., Barnes \& O'Brien 2002; Spalding et al. 2016). Recent large-scale efforts have broadly applied new techniques to identify exomoon host candidates in data from transit surveys, including Kepler (Kipping \& Teachey 2020; Rodenbeck et al. 2020). Now that the long-period giant planet Kepler-1514b has had its mass measured, the Kepler-1514 system is likely worth revisiting for a more focused investigation of the possible existence and detectability of exomoon candidates.

\section{Summary}

We conducted RV observations of Kepler-1514 using the HIRES instrument on the Keck I telescope. Based on data collected by the primary Kepler mission (Borucki et al. 2010) and analysis conducted by Morton et al. (2016), this system was thought to contain a cool gas giant planet on a 218-day orbital period (that was statistically validated) and a shorterperiod Earth-size KOI. The transits of each object in the Kepler-1514 system displayed variations in timing (relative to a linear ephemeris), depth, and duration (Holczer et al. 2016). Inspired by the high false-positive probability of long-period $(P \gtrsim 100$ days), giant planet signals in Kepler transit data (Santerne et al. 2016) and also by the inherent rarity of longperiod transiting exoplanets, we aim to measure the mass of Kepler-1514 b and characterize the system.

We apply spline detrending to remove the stellar variability of the host star present in the Kepler photometry (Section 3.1). This detrending casts doubt on a dynamical explanation for the TTVs and T $\delta$ Vs (see Section 3), but we nonetheless include the former in the comprehensive global modeling of the transit and RV data. The RV observations (Section 2.2) readily identify a planetary, Keplerian signal corresponding to Kepler-1514 b, which we find to be massive $\left(M_{p}=5.28 \pm 0.22 M_{\mathrm{J}}\right)$ and on a moderately eccentric orbit $\left(e=0.401_{-0.014}^{+0.013}\right)$. The modest set of $\mathrm{RVs}$, although precise, is not able to constrain the mass of KOI3681.02, for which we expect a sub-meter-per-second RV semiamplitude. However, through a false-positive probability analysis that includes scenarios introduced by neighboring stars, we validate the planetary nature of KOI-3681.02 (now known as Kepler-1514c) with a false-positive probability below $1 \%$ (Section 4.2).

Based on these results, we postulate on the possible interior properties and formation history of Kepler-1514 b and its utility as one of only a select few long-period ( $P>100$ days) giant exoplanets with a well-known mass and radius (Section 5.2). Kepler-1514 b is unlikely to be inflated (e.g., Demory \& Seager 2011; Miller \& Fortney 2011) like its hot Jupiter counterparts, but its relatively high mass makes it a useful test of the radius inflation thresholds put forth by Sestovic et al. (2018). Based on the lack of a known associated stellar companion (Section 2.3), we assert that Kepler-1514 b may have migrated via planet-planet scattering, although we cannot rule out other mechanisms. The high bulk density Kepler$1514 \mathrm{~b}\left(4.82_{-0.25}^{+0.26} \mathrm{~g} \mathrm{~cm}^{-3}\right)$ is atypical among giant planets but is consistent with those having nearly constant radius above $\sim 0.5 M_{\mathrm{J}}$ masses because of electron degeneracy pressure.

Moving forward, we consider Kepler-1514 b as a candidate for further investigation (Section 5.3). Although prospects for atmospheric characterization via transmission spectroscopy are poor, the system is highly amenable to a stellar obliquity measurement via the RM effect. Furthermore, Kepler-1514 b has been previously identified as a promising system for searches for exomoons. With the new mass measurement presented here, we recommend a focused reexamination of the Kepler-1514 system and its potential to harbor natural satellites.

We note that, during the preparation of this manuscript, KOI3681.02 was statistically validated as Kepler-1514 c by Armstrong et al. (2020).

The authors thank the anonymous referee for thoughtful comments that improved the quality and clarity of this work. The authors thank all of the observers in the California Planet Search team for their many hours of hard work. P.D. is supported by a National Science Foundation (NSF) Astronomy and Astrophysics Postdoctoral Fellowship under award AST-1903811. This research has made use of the NASA Exoplanet Archive, which is operated by the California Institute of Technology, under contract with the National Aeronautics and Space Administration under the Exoplanet Exploration Program. This research made use of exoplanet and its dependencies (Kipping 2013; Astropy Collaboration et al. 2013, 2018; Salvatier et al. 2016; Theano Development Team 2016; Luger et al. 2019; Agol et al. 2020).

This paper includes data collected by the Kepler mission and obtained from the MAST data archive at the Space Telescope Science Institute (STScI). Funding for the Kepler mission is provided by the NASA Science Mission Directorate. STScI is operated by the Association of Universities for Research in Astronomy, Inc., under NASA contract NAS 5-26555. Some of the data presented herein were obtained at the W. M. Keck Observatory, which is operated as a scientific partnership among the California Institute of Technology, the University of California, and NASA. The Observatory was made possible by the generous financial support of the W. M. Keck Foundation. Finally, the authors wish to recognize and acknowledge the very significant cultural role and reverence that the summit of Maunakea has always had within the indigenous Hawaiian community. We are most fortunate to have the opportunity to conduct observations from this mountain.

Facilities: Keck:I (HIRES), Keck:II (NIRC2), Kepler.

Software: astropy (Astropy Collaboration et al. 2013, 2018), EXOFASTv2 (Eastman et al. 2013; Eastman 2017; Eastman et al. 2019), VESPA (Morton 2012, 2015), exoplanet (ForemanMackey et al. 2020), pymc3 (Salvatier et al. 2016), theano, (Theano Development Team 2016). 


\section{ORCID iDs}

Paul A. Dalba (1) https://orcid.org/0000-0002-4297-5506 Stephen R. Kane (1) https://orcid.org/0000-0002-7084-0529 Howard Isaacson (1) https://orcid.org/0000-0002-0531-1073 Steven Giacalone (i) https://orcid.org/0000-0002-8965-3969 Andrew W. Howard (ㄱ) https://orcid.org/0000-00018638-0320

Joseph E. Rodriguez iㅏ https://orcid.org/0000-00018812-0565

Andrew Vanderburg (1) https://orcid.org/0000-00017246-5438

Jason D. Eastman (i) https://orcid.org/0000-0003-3773-5142 Adam L. Kraus (i) https://orcid.org/0000-0001-9811-568X Trent J. Dupuy (ㄴ) https://orcid.org/0000-0001-9823-1445 Lauren M. Weiss (ib https://orcid.org/0000-0002-3725-3058 Edward W. Schwieterman (1) https://orcid.org/0000-00022949-2163

\section{References}

Agol, E., Luger, R., \& Foreman-Mackey, D. 2020, AJ, 159, 123 Alonso, R., Auvergne, M., Baglin, A., et al. 2008, A\&A, 482, L21 Alp, D., \& Demory, B. O. 2018, A\&A, 609, A90

Armstrong, D. J., Gamper, J., \& Damoulas, T. 2020, arXiv:2008.10516 Astropy Collaboration, Price-Whelan, A. M., Sipőcz, B. M., et al. 2018, AJ, 156,123

Astropy Collaboration, Robitaille, T. P., Tollerud, E. J., et al. 2013, A\&A, 558, A33

Barnes, J. W., \& O’Brien, D. P. 2002, ApJ, 575, 1087

Beatty, T. G., \& Gaudi, B. S. 2008, ApJ, 686, 1302

Bonomo, A. S., Desidera, S., Benatti, S., et al. 2017, A\&A, 602, A107

Borucki, W. J., Koch, D., Basri, G., et al. 2010, Sci, 327, 977

Brahm, R., Jordán, A., Bakos, G. A., et al. 2016, AJ, 151, 89

Bryan, M. L., Knutson, H. A., Lee, E. J., et al. 2019, AJ, 157, 52

Chatterjee, S., Ford, E. B., Matsumura, S., \& Rasio, F. A. 2008, ApJ, 686, 580

Chen, J., \& Kipping, D. 2017, ApJ, 834, 17

Choi, J., Dotter, A., Conroy, C., et al. 2016, ApJ, 823, 102

Cooke, B. F., Pollacco, D., Anderson, D. R., et al. 2021, MNRAS, 500, 5088

Cooke, B. F., Pollacco, D., West, R., McCormac, J., \& Wheatley, P. J. 2018, A\&A, 619, A175

Dalba, P. A. 2017, ApJ, 848, 91

Dalba, P. A., Fulton, B., Isaacson, H., Kane, S. R., \& Howard, A. W. 2020a, AJ, 160, 149

Dalba, P. A., Gupta, A. F., Rodriguez, J. E., et al. 2020b, AJ, 159, 241

Dalba, P. A., Kane, S. R., Barclay, T., et al. 2019, PASP, 131, 034401

Dalba, P. A., \& Muirhead, P. S. 2016, ApJL, 826, L7

Dalba, P. A., Muirhead, P. S., Croll, B., \& Kempton, E. M.-R. 2017, AJ, 153,59

Dalba, P. A., Muirhead, P. S., Fortney, J. J., et al. 2015, ApJ, 814, 154

Dalba, P. A., \& Tamburo, P. 2019, ApJL, 873, L17

Dawson, R. I., \& Johnson, J. A. 2018, ARA\&A, 56, 175

Debras, F., Baruteau, C., \& Donati, J.-F. 2021, MNRAS, 500, 1621

Deming, L. D., \& Seager, S. 2017, JGRE, 122, 53

Demory, B.-O., \& Seager, S. 2011, ApJS, 197, 12

Díaz, M. R., Jenkins, J. S., Feng, F., et al. 2020, MNRAS, 496, 4330

Dittmann, J. A., Irwin, J. M., Charbonneau, D., et al. 2017, Natur, 544, 333

Dotter, A. 2016, ApJS, 222, 8

Dubber, S. C., Mortier, A., Rice, K., et al. 2019, MNRAS, 490, 5103

Dupuy, T. J., Kratter, K. M., Kraus, A. L., et al. 2016, ApJ, 817, 80

Eastman, J. 2017, EXOFASTv2: Generalized publication-quality exoplanet modeling code, v2, Astrophysics Source Code Library, ascl:1710.003

Eastman, J., Gaudi, B. S., \& Agol, E. 2013, PASP, 125, 83

Eastman, J. D., Rodriguez, J. E., Agol, E., et al. 2019, arXiv:1907.09480

Eisner, N. L., Barragán, O., Aigrain, S., et al. 2020, MNRAS, 494, 750

Fabrycky, D., \& Tremaine, S. 2007, ApJ, 669, 1298

Ford, E. B. 2006, ApJ, 642, 505

Foreman-Mackey, D., Luger, R., Czekala, I., et al. 2020, exoplanet-dev/ exoplanet v0.3.2, Zenodo, doi:10.5281/zenodo.1998447

Foreman-Mackey, D., Morton, T. D., Hogg, D. W., Agol, E., \& Schölkopf, B. 2016, AJ, 152, 206

Fortney, J. J., Marley, M. S., \& Barnes, J. W. 2007, ApJ, 659, 1661
Fulton, B. J., Collins, K. A., Gaudi, B. S., et al. 2015, ApJ, 810, 30 Furlan, E., Ciardi, D. R., Everett, M. E., et al. 2017, AJ, 153, 71

Gaia Collaboration, Brown, A. G. A., Vallenari, A., et al. 2018, A\&A, 616, A1 Gelman, A., \& Rubin, D. B. 1992, StaSc, 7, 457

Giles, H. A. C., Osborn, H. P., Blanco-Cuaresma, S., et al. 2018, A\&A, $615, \mathrm{~L} 13$

Gill, S., Wheatley, P. J., Cooke, B. F., et al. 2020, ApJL, 898, L11

Girardi, L., Groenewegen, M. A. T., Hatziminaoglou, E., \& da Costa, L. 2005, A\&A, 436, 895

Groenewegen, M. A. T., Girardi, L., Hatziminaoglou, E., et al. 2002, A\&A, 392,741

Guimarães, A., \& Valio, A. 2018, AJ, 156, 50

Herman, M. K., Zhu, W., \& Wu, Y. 2019, AJ, 157, 248

Hill, M. L., Kane, S. R., Seperuelo Duarte, E., et al. 2018, ApJ, 860, 67

Holczer, T., Mazeh, T., Nachmani, G., et al. 2016, ApJS, 225, 9

Howard, A. W., \& Fulton, B. J. 2016, PASP, 128, 114401

Howard, A. W., Johnson, J. A., Marcy, G. W., et al. 2010, ApJ, 721, 1467

Howell, S. B., Sobeck, C., Haas, M., et al. 2014, PASP, 126, 398

Irwin, P. G. J., Barstow, J. K., Bowles, N. E., et al. 2014, Icar, 242, 172

Isaacson, H., \& Fischer, D. 2010, ApJ, 725, 875

Jenkins, J. M., Caldwell, D. A., Chandrasekaran, H., et al. 2010, ApJL, 713, L87

Kawahara, H., \& Masuda, K. 2019, AJ, 157, 218

Kinemuchi, K., Barclay, T., Fanelli, M., et al. 2012, PASP, 124, 963

Kipping, D., \& Teachey, A. 2020, SerAJ, 201, 25

Kipping, D. M. 2009a, MNRAS, 392, 181

Kipping, D. M. 2009b, MNRAS, 396, 1797

Kipping, D. M. 2013, MNRAS, 435, 2152

Kipping, D. M., Bakos, G. Á., Buchhave, L., Nesvorný, D., \& Schmitt, A. 2012, ApJ, 750, 115

Kipping, D. M., Schmitt, A. R., Huang, X., et al. 2015, ApJ, 813, 14

Kolbl, R., Marcy, G. W., Isaacson, H., \& Howard, A. W. 2015, AJ, 149, 18

Kraus, A. L., Ireland, M. J., Huber, D., Mann, A. W., \& Dupuy, T. J. 2016, AJ, 152,8

Laughlin, G., Deming, D., Langton, J., et al. 2009, Natur, 457, 562

Lendl, M., Bouchy, F., Gill, S., et al. 2020, MNRAS, 492, 1761

Lissauer, J. J., Marcy, G. W., Rowe, J. F., et al. 2012, ApJ, 750, 112

Lucy, L. B., \& Sweeney, M. A. 1971, AJ, 76, 544

Luger, R., Agol, E., Foreman-Mackey, D., et al. 2019, AJ, 157, 64

Madhusudhan, N. 2019, ARA\&A, 57, 617

Mandel, K., \& Agol, E. 2002, ApJL, 580, L171

Markwardt, C. B. 2009, in ASP Conf. Ser. 411, Astronomical Data Analysis Software and Systems XVIII, ed. D. A. Bohlender, D. Durand, \& P. Dowler (San Francisco, CA: ASP), 251

Masuda, K., Winn, J. N., \& Kawahara, H. 2020, AJ, 159, 38

McLaughlin, D. B. 1924, ApJ, 60, 22

Miller, N., \& Fortney, J. J. 2011, ApJL, 736, L29

Morton, T. D. 2012, ApJ, 761, 6

Morton, T. D. 2015, VESPA: False Positive Probabilities Calculator, Astrophysics Source Code Library, ascl:1503.011

Morton, T. D., Bryson, S. T., Coughlin, J. L., et al. 2016, ApJ, 822, 86

Moutou, C., Hébrard, G., Bouchy, F., et al. 2009, A\&A, 498, L5

Naef, D., Latham, D. W., Mayor, M., et al. 2001, A\&A, 375, L27

Osborn, H. P., Armstrong, D. J., Brown, D. J. A., et al. 2016, MNRAS, 457,2273

Oshagh, M., Santos, N. C., Boisse, I., et al. 2013, A\&A, 556, A19

Paxton, B., Bildsten, L., Dotter, A., et al. 2011, ApJS, 192, 3

Paxton, B., Cantiello, M., Arras, P., et al. 2013, ApJS, 208, 4

Paxton, B., Marchant, P., Schwab, J., et al. 2015, ApJS, 220, 15

Petigura, E. A., Sinukoff, E., Lopez, E. D., et al. 2017, AJ, 153, 142

Ricker, G. R., Winn, J. N., Vanderspek, R., et al. 2015, JATIS, 1, 014003

Rodenbeck, K., Heller, R., \& Gizon, L. 2020, A\&A, 638, A43

Rossiter, R. A. 1924, ApJ, 60, 15

Salvatier, J., Wiecki, T. V., \& Fonnesbeck, C. 2016, PeerJ Computer Science, 2, e55

Santerne, A., Moutou, C., Tsantaki, M., et al. 2016, A\&A, 587, A64

Sartoretti, P., \& Schneider, J. 1999, A\&AS, 134, 553

Schlegel, D. J., Finkbeiner, D. P., \& Davis, M. 1998, ApJ, 500, 525

Seager, S., \& Mallén-Ornelas, G. 2003, ApJ, 585, 1038

Seager, S., \& Sasselov, D. D. 2000, ApJ, 537, 916

Sestovic, M., Demory, B.-O., \& Queloz, D. 2018, A\&A, 616, A76

Sidis, O., \& Sari, R. 2010, ApJ, 720, 904

Simon, A., Szatmáry, K., \& Szabó, G. M. 2007, A\&A, 470, 727

Sing, D. K., Fortney, J. J., Nikolov, N., et al. 2016, Natur, 529, 59

Smith, J. C., Stumpe, M. C., Van Cleve, J. E., et al. 2012, PASP, 124, 1000

Socia, Q. J., Welsh, W. F., Orosz, J. A., et al. 2020, AJ, 159, 94 
Spalding, C., Batygin, K., \& Adams, F. C. 2016, ApJ, 817, 18

Stassun, K. G., \& Torres, G. 2018, ApJ, 862, 61

Stumpe, M. C., Smith, J. C., Van Cleve, J. E., et al. 2012, PASP, 124, 985

Szabó, G. M., Szatmáry, K., Divéki, Z., \& Simon, A. 2006, A\&A, 450, 395

Tayar, J., Claytor, Z. R., Huber, D., \& van Saders, J. 2020, arXiv:2012.07957

Teachey, A., Kipping, D. M., \& Schmitt, A. R. 2018, AJ, 155, 36

Theano Development Team 2016, arXiv:abs/1605.02688

Thompson, S. E., Coughlin, J. L., Hoffman, K., et al. 2018, ApJS, 235, 38

Thorngren, D. P., Fortney, J. J., Murray-Clay, R. A., \& Lopez, E. D. 2016, ApJ, 831, 64

Triaud, A. H. M. J. 2018, in Handbook of Exoplanets, ed. H. Deeg \& J. Belmonte (Cham: Springer), 2

Uehara, S., Kawahara, H., Masuda, K., Yamada, S., \& Aizawa, M. 2016, ApJ, 822,2

Vanderburg, A., Becker, J. C., Kristiansen, M. H., et al. 2016a, ApJL, 827, L10

Vanderburg, A., Huang, C. X., Rodriguez, J. E., et al. 2019, ApJL, 881, L19

Vanderburg, A., Latham, D. W., Buchhave, L. A., et al. 2016b, ApJS, 222, 14
Vanhollebeke, E., Groenewegen, M. A. T., \& Girardi, L. 2009, A\&A, 498, 95 Villanueva, S., J., Dragomir, D., \& Gaudi, B. S. 2019, AJ, 157, 84

Vogt, S. S., Allen, S. L., Bigelow, B. C., et al. 1994, Proc. SPIE, 2198, 362

Wakeford, H. R., Sing, D. K., Kataria, T., et al. 2017, Sci, 356, 628

Wang, J., Fischer, D. A., Barclay, T., et al. 2015, ApJ, 815, 127

Weiss, L. M., Marcy, G. W., Rowe, J. F., et al. 2013, ApJ, 768, 14

Welbanks, L., Madhusudhan, N., Allard, N. F., et al. 2019, ApJL, 887, L20

Welsh, W. F., \& Orosz, J. A. 2018, in Handbook of Exoplanets, ed. H. Deeg \& J. Belmonte (Cham: Springer), 34

Winn, J. N. 2010, in Exoplanets, ed. S. Seager (Tucson, AZ: Univ. Arizona Press), 55

Winn, J. N., Albrecht, S., Johnson, J. A., et al. 2011, ApJL, 741, L1

Wizinowich, P., Acton, D. S., Shelton, C., et al. 2000, PASP, 112, 315

Yee, S. W., Petigura, E. A., \& von Braun, K. 2017, ApJ, 836, 77

Yelda, S., Lu, J. R., Ghez, A. M., et al. 2010, ApJ, 725, 331

Zapolsky, H. S., \& Salpeter, E. E. 1969, ApJ, 158, 809

Zhu, W., \& Wu, Y. 2018, AJ, 156, 92 